\title{
AN AUTOMATIC SEGMENTATION OF LIVER COLUME THAT CONTAINED DISCONNECTED REGION WITH DYNAMIC LOCAL NEIGHBOURHOOD REGION SIZES
}

\author{
Puteri Suhaiza Sulaiman ${ }^{1}$, Rahmita Wirza Rahmat ${ }^{1}$, Ramlan Mahmod ${ }^{1}$, Abdul Hamid Abdul \\ Rashid $^{2}$ \\ ${ }^{1}$ Department Of Multimedia, FSKTM \\ Universiti Putra Malaysia, \\ 43400 Serdang, Selangor \\ \{suhaiza, rahmita, ramlan\}@fsktm.upm.edu.my \\ ${ }^{2}$ Department Of Anatomy, FPSK \\ Universiti Putra Malaysia, \\ 43400 Serdang, Selangor \\ dahar@medic.upm.edu.my
}

\begin{abstract}
Segmentation of liver images containing disconnected regions has always been an overlooked problem. Previous works on liver segmentation either ignore this problem or use manual initialization when facing these disconnected regions. Therefore, in this paper we propose a liver level set (LLS) algorithm which is able to segment disconnected regions automatically. The LLS algorithm is based on level set framework together with hybrid energy minimization as the stopping function. By using the LLS algorithm in a looping manner, we allow the current liver boundary to inherit the topological changes from previous images in a 2.5D environment. We also conduct an experiment to obtain an average factor for dynamic localization region sizes based on liver anatomy to improve the segmentation accuracy. These dynamic localization region sizes ensure a more accurate segmentation when compared with manual segmentation. Our experiment gives a respective segmentation result with dice similarity coefficient (DSC) percentage of $\mathbf{8 7 . 5 \%}$. Plus, our LLS algorithm is able to segment all connected and disconnected liver region automatically and accurately.
\end{abstract}

Keywords: Liver segmentation, level set algorithm, liver disconnected regions.

\section{INTRODUCTION}

The aim in liver segmentation process is to extract liver volume accurately and automatically in less time as possible. Notably the liver has been considered as the most difficult organ to segment in abdominal area and even with the assistance of radiologists, extracting liver contour from each slice is still a daunting tasks. The intricacy for this segmentation process is cause by the liver's form itself, since liver is made from soft tissues, there a large variation of liver geometry between patients. Plus, liver has similar tissues density with neighbouring organ such as the heart and kidney, causing a limited contrast in CT grey level between them.

Another frequently overlooked feature of liver's formic that the liver has a multi-lobe structure, thus different lobes can exhibit as disconnected regions in a transverse slice. Hence in the worst case scenario, liver may have more than two disconnected regions with variation of shape in a single CT image as shown in Figure 1. Currently, there still lack of on paper solution for extracting these disconnected regions automatically. Usually, some user interference still needed when segmenting these CT liver images.

This paper is organized as follows. In section 2, several researches on liver segmentation are reviewed. Section 3 reveals our proposed LLS algorithms in details. In section 4, we discuss the experiments and result on the LLS algorithm. Finally in section 5, we present our concluding remarks, together with the benefits, drawbacks and future works. 

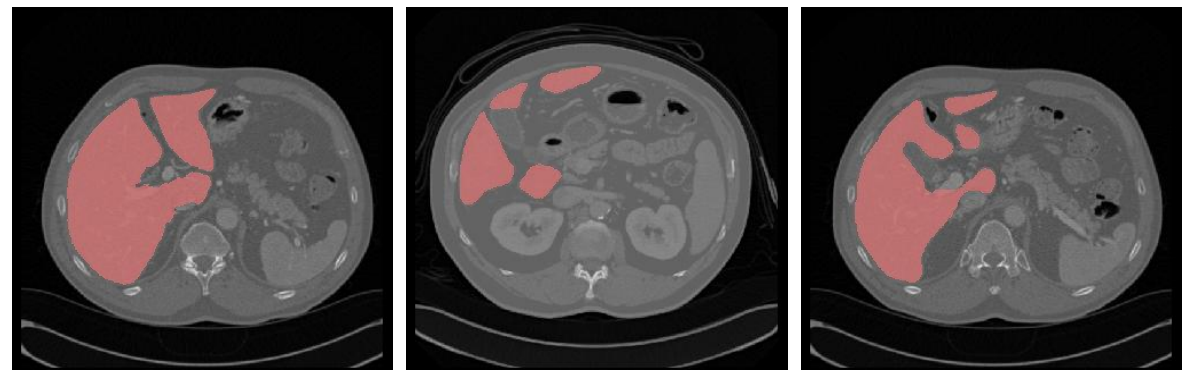

Figure 1: The disconnected regions are highlighted with red region

\section{RELATED WORKS}

Liver segmentation is a process of extracting liver region from abdominal CT scan images. The output for liver segmentation is the binary mask, where the extracted liver region is rendered as white with a black background. As mentioned earlier, the liver segmentation is a very intricate process, where the process typically remains semi-automatic or interactive, with user guidance needed in order to correctly help in identification. Segmentation process itself is a major field of study; a useful survey paper that can be served as the basis for medical image segmentation is given by Pham and et al. (2000). They suggested that a typical strategy in medical image segmentation is to start with simple techniques initially, and seek a more supplicated technique if these techniques fail. Plus, a fusion between several techniques can also helped in the segmenting process of complicated organ such as the liver and tumours.

Earlier works on liver segmentation are based on grey level image processing operation such as histogram analysis, thresholding, morphological operators and active (Bae et al., 1993; Kobashi \& Shapiro, 1995; Gao et al., 1998). Generally, the first step is to estimate the liver grey value either by using histogram analysis in a grey level range or by statistical analysis of several segmented liver slices. Next, simple or iterative thresholding technique is used to create a series of binary images, in which will then refines using 2D or 3D morphological operation. The last step is smoothing these boundaries in each slice by means of B-splines, or using the active contours also known as SNAKE (Kass et al., 1988).

A more recent works by Lim are also basing on the tree steps mention above (Lim et al., 2004; Lim et al., 2006; Lim et al., 2005). They estimates the liver grey value by analyzing the intensities distribution on a subset of manually segmented lever images together with prior knowledge of liver location. Using both the information, a simple thresholding is done to create a coarse liver volume. Then, an interactive morphological filtering and k-means clustering are then used to find and delete neighbouring organ attached to the liver and define a search region where the final liver boundary is determined. Finally, an active contour with minimum cost function is used to refine the liver boundary. This algorithm has an expensive processing time which takes about 1-3 min/slice on Pentium 4 3.0 GHz system. The authors evaluate their system on several samples with various shapes and irregular texture of 10 patients. The results are evaluated by comparing with the ground truth produce by experts, and the $4 \%$ of mismatching voxels are obtained.

Another combination of histogram analysis, thresholding, morphological operators and active contour combination are done by Liu et al. (2005). They estimate the liver grey value by looking at the histogram of the whole volume and identify two liver thresholds, one at each side of the liver peaks. These thresholds are employed to create a liver binary volume, which then processes through a series of morphological operation. The processed binary volume is used to create a liver mask using Canny edge detector. The edge of this liver mask is used as the input for the gradient vector flow algorithm. To refine the liver boundary, user has to select a starting slice which has a large area profile and most accurate segmentation result. This selected slice will be used as a mask to detect and eliminated errors of its adjacent slices. This iterative correction process is done in a slice by slice manner.

To our knowledge, only Areste et al. (2005) directly deal with liver disconnected region by doing a 3D region growing algorithm to obtain an initial segmentation of the liver. This algorithm starts with a set of seed points and grows from it in a repeated fashion by appending to each seed of its neighbouring voxel that have similar intensities values. In order to ensure a successful segmentation of different lobes of the liver, a wide intensities threshold range is used. This has caused the segmentation result to include artefacts from neighbouring tissues. A $3 \mathrm{D}$ erosion operation and $2 \mathrm{~d}$ region growing algorithm are then applied for each slice to further refine the liver contour. A major disadvantage of Areste's technique is that it involved 2 layers of region growing algorithms, which can be both very consuming in processing time and memory.

In all the grey operation techniques mentioned above, the successfulness of their systems relies on the grey value estimation. Therefore, finding the most accurate threshold values defining the liver were very important 
and selecting the wrong values might cause a disconnected region to be removed. Plus the use of morphological filtering might reject some separated liver regions. Thus relaying solely on threshold value, morphological operators and region growing are not suitable for segmenting liver with several disconnected regions automatically.

Other noticeable works are based on the construction of probabilistic atlases for abdomen organ done by Zhou et al. (2005) and Shimizu et al. (2007). The first step to build the probabilistic atlas is the registration of each training CT data into a standard space defined by a small set of landmarks. The thin plate spline is used as the warping transform, together with the mutual information as the similarity measure. The probabilistic atlas is then created by spatial averaging the registered organ surfaces, and it is incorporated into a Bayesian framework to compute, for each voxel, the probability of belonging to a certain organ. Finally, the region that maximizes the posterior probability of being the desired organ is extracted with the iterative conditional mode algorithm, or by simple thresholding. Shimizu et al. (2005) and Zhou et al. (2005) give a respective 78\% and 84\% mean 3D volume overlap, between the automatic and manual liver segmentation. Park presented their results by measuring the average percentage of mismatching pixels between the automatic and manual segmentation, and obtained $8.5 \%$ error rate.

Another similar approach is introduced by Lamecker et al. (2004) by introducing a 3D statistical shape models for robust and automatic segmentation of medical images. They generate a statistical shape model from a training set that consist of $30 \mathrm{CT}$ scans with contrast enhancement and a gap of $5 \mathrm{~mm}$ between two consecutive slices. However the result is not satisfying due to the fact that there is a variation in the liver shape and scale across patient even on the same patient.

The difficulty with probabilistic atlases and statistical shape models methods are the requirements of huge sum of sample and training data. Considering the fact that liver is made from soft tissues, therefore there was a large variation of liver shape between patients has become the main obstacle for both methods. The result may valid for liver with standard shape but when dealing with liver with cancer that contains large tumours, the shape of liver usually mutated, therefore resulting wrong segmentation.

A completely different approach to segment the liver was by using level set algorithm. First attempt to segment the liver using level set is done by Pan and Dawant (2001). They introduces an accumulative level-set speed function which varied by time to improve the detection sensitivity of weak edges. Plus, they also incorporate prior liver location based on anatomy knowledge to help in the segmentation process. Pan's 2D algorithm begins by initializing the curve through putting a small circle inside the liver region for each slice. Thus, if a disconnected region occurs in the current slice, a user needs to initialize a circle for each disconnected regions separately.

Lee and colleagues (2007) advances the level set by predicting the initial liver shape using 2.5D shape propagation. So instead of placing the curve in each slice, Lee's method required a manual input of seed points for each of the topmost and the bottommost liver slice. And if there are regions that are not connected from previous slice, additional seed points are needed.

Both attempts of using level set approach for liver segmentation required additional user interaction for disconnected regions (Pan et al., 2001; Lee et al., 2007). This does not fully utilize the benefits for using levels sets which able to carter the merging and separating propagating front. Sulaiman and colleagues (2008) experimented using level set and hybrid energy minimizing stopping function for extracting liver with disconnected regions. They uses the hybrid energy minimizing stopping function proposed by Lankton et al. (2007), to ensures that the liver regions are segmented accurately despite of homogeneous background. Thus, this paper is an extension to Sulaiman et al.'s work (2008) where we propose a dynamic local neighbourhood sizes to improve the overall segmentation result.

\section{METHOD}

This section will describe in details the liver level set algorithm. There are three main elements that contribute to LLS algorithm which are the initial curve, the size of liver local neighbourhood and the hybrid energy minimization algorithm. All these elements are combined in a looping manner for the automatic LLS algorithm.

\subsection{Initial Curve Location}

In level set function, location of the initial curve is very important. Previously reviewed works on level set requires the placement of initial curve to be inside the liver boundary and additional placement of another initial curve for disconnected region. Since the CT liver datasets consists of a series of connected slice images which are closely related to each other, an assumption is made, that a liver boundary in a slice is slightly different from its adjacent slice. This allows the use of the segmented liver boundary from the previous slice as the initial curve for the next liver boundary to be segmented as in $2.5 \mathrm{D}$ environment. The advantage of doing this is to allow a smooth flow of boundary information between the slice images thus, making it possible to segment 
disconnected region automatically. Plus, since we basing the LLS algorithm using the energy minimization, the initial curve can be place inside or outside the liver boundary, thus allowing the curve to propagate by expanding and shrinking towards the liver boundary.

\subsection{Liver Local Neighbourhood Region}

The successfulness of our purpose algorithm depends on the size of the liver local neighbourhood. This is because, if the local neighbourhood is too small, then it will fail to capture the entire liver region. Likewise, if the local neighbourhood is too big, then it will include several nearby artefacts. In the Lankton's algorithm, the local neighbourhood remains as a constant, and it is up to the user to find the best size for the local neighbourhood depending on the image to be segment (Lankton et al., 2007). Thus, in our case, we need to find the size of local neighbourhood for liver regions that allows a more robust segmentation algorithm. Therefore, we have conducted further study on liver images to find a generalization factor.

A study is conducted on $20 \mathrm{CT}$ scan datasets, in which each of them come from various patient and condition. Each image in the datasets is in the same size of 512X512 but has different thickness. Further study on these datasets, we find that a smaller local neighbourhood region is needed in the middle of these liver images, because these middle images contain a lot of nearby adjacent organ that might contribute to the means intensities of the local region calculation. However, a larger local neighbourhood region is needed in the starting and ending slice to completely capture remaining liver region. From this we have made the assumption that a series of liver images can be divided into three stages as shown in Figure 2.

Stage 1 begins when the liver region start to appears in the image series. We tag the first slice as SLN (start liver number). Stage 2 begins when the portal veins began to leave the liver boundary, and we tag the first slice for stage 2 as $\mathrm{SmN}$ (start middle number). During this stage, liver boundary near the portal veins is closely surrounded by gallbladder and stomach. Stage 2 ends when the liver boundary starts to combined back together, completely contained the portal veins inside. We mark the end slice as EmN (end middle number). Next slice after EmN is the starting slice for stage 3. The end slice for stage 3 will be marked as ELN (end liver number). Based on the above assumption we manually tag SLN, ELN, SmN and EmN for each patient in our sample dataset.

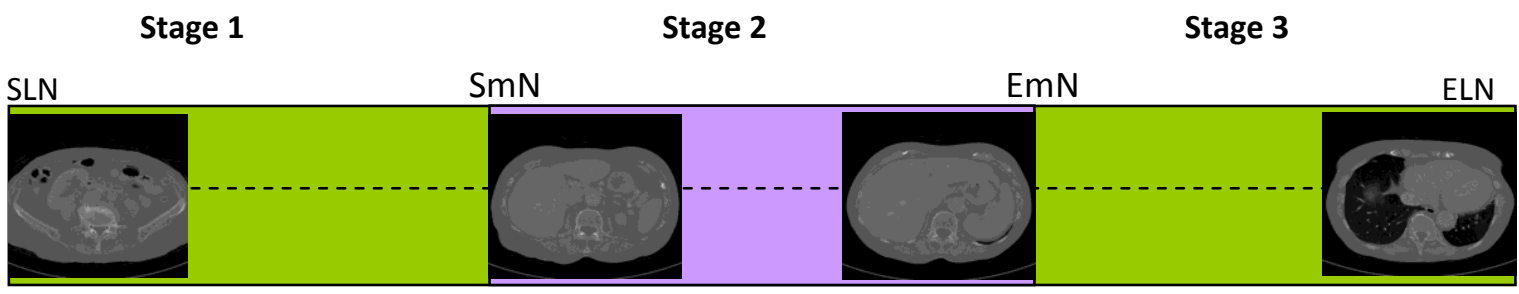

Figure 2: The assumption of CT liver stages

By giving the same constant $\beta$ value for stage 1 and stage 3 , and a constant $\delta$ value for stage 2 , we generate a graph as shown in Figure 3 below. From this graph we find that the overall distribution of all 20 patients is varies in the x position depending on the CT thickness. Therefore to generate the general pattern, we have normalized the distribution so that the value of SLN and ELN are 0 and 100 respectively. Then we calculate the average value of $\mathrm{SmN}$ and $\mathrm{SmE}$ to get the value generalization factor of Gfs and Gfe.

By using these Gfs and Gfe value and the input value of SmN and EmN, we can obtain SmN and EmN automatically using the following equations

$$
\begin{aligned}
& S m N=\left|\left(\frac{G f S^{*}(E L N-S L N}{100}\right)+S L N\right| \\
& E m N=\left|\left(\frac{G f e^{*}(E L N-S L N}{100}\right)+S L N\right|
\end{aligned}
$$




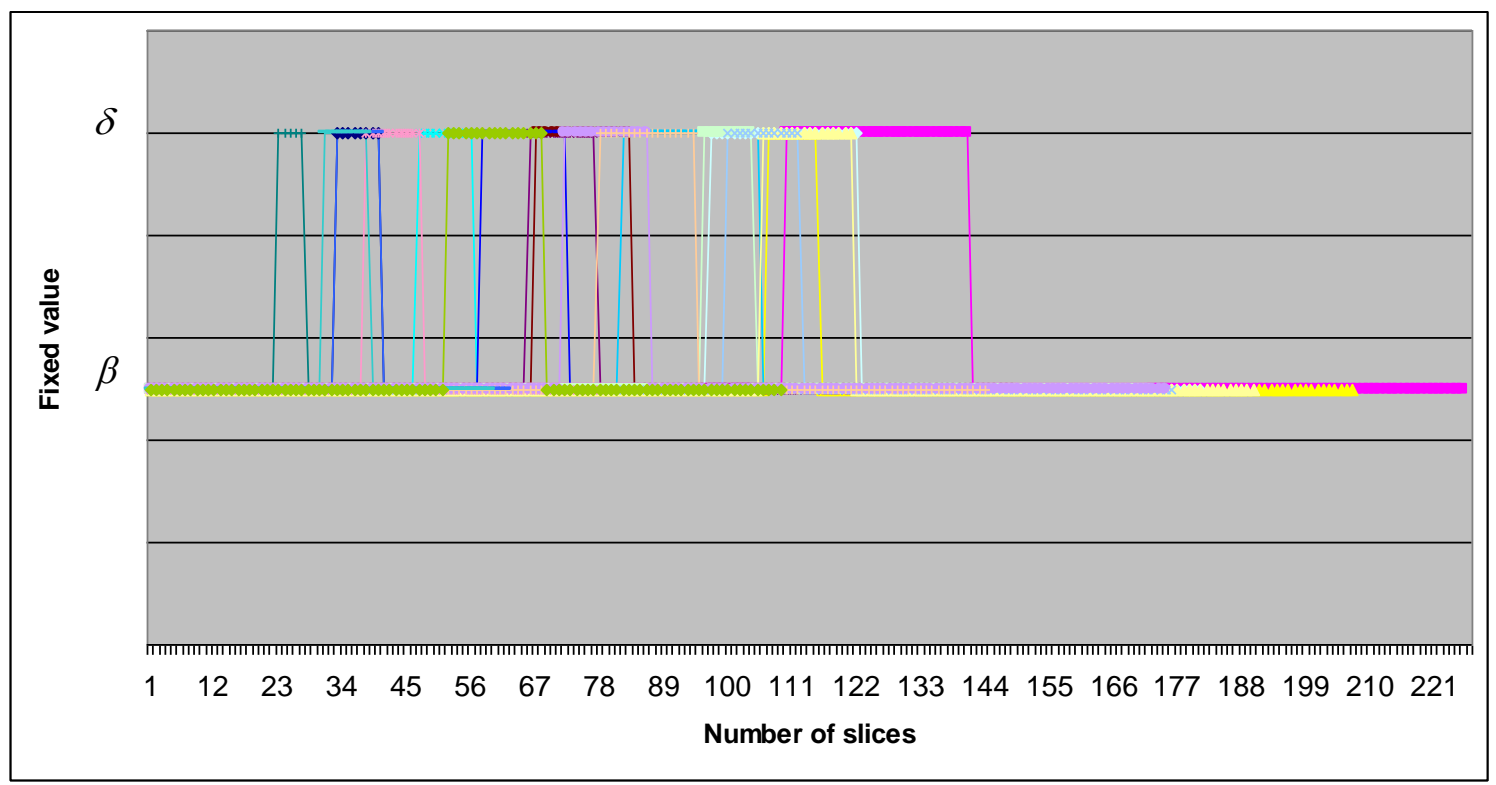

Figure 3: 20 patients with a fixed local neighbourhood size of $\beta$ and $\delta$

\subsection{Level Set with Hybrid Energy Based Function}

The next steps is embedded the idea of liver local neighbourhood region in level set function framework. We start with the hybrid energy proposed by Lankton et al. (2007),

$$
E=\oint_{C(s)} \int_{x \in \omega}\left(I \chi(x, s)-(u \ell(s))^{2}+\int_{x \in \omega}(I \chi(x, s)-v \ell(s))^{2} d s\right.
$$

where $u \ell(s)$ and $\nu \ell(s)$ are the arithmetic means of points in local neighbourhoods around the points, $C(s)$. A given image I defined on the domain $\Omega$, and $\mathrm{C}$ represents closed contour represented as the zero level set of a signed distance function. The interior region $\omega$ of $\mathrm{C}$ is defined by the following approximation of the smoothed Heaviside function:

$\mathrm{H} \phi(x)=\left\{\begin{array}{cc}1, & \phi(x)<-\varepsilon \\ 0, & \phi(x)>\varepsilon \\ \frac{1}{2}\left\{1+\frac{\phi}{\varepsilon}+\frac{1}{2} \sin \left(\frac{\pi \phi(x)}{\varepsilon}\right)\right\}, & \text { otherwise }\end{array}\right.$

Likewise, the exterior region $\varpi$ is defined as $(1-\mathbf{H} \phi(x))$. The derivative of $\mathrm{H} \phi(x)$ is used to specify the area just around the curve and can be presents as a smoothed version of the Dirac delta,

$$
\delta \phi(x)=\left\{\begin{array}{cc}
1, & \phi(x)=0 \\
0, & |\phi(x)|<\varepsilon \\
\frac{1}{2 \varepsilon}\left\{1+\cos \left(\frac{\pi \phi(x)}{\varepsilon}\right)\right\}, & \text { otherwise }
\end{array}\right.
$$


The mask for local region is a circle given by the following equation;

$$
B(x, y)= \begin{cases}1, & \|x-y\|<r \\ 0, & \text { otherwise }\end{cases}
$$

in which $x$ and $y$ represents independent spatial variables each representing a single point on $\Omega$. This function will be 1 when point $y$ is within a ball of radius $\mathrm{r}$ centred at $x$, and 0 otherwise. Using the $B(x, y)$, energy functional in term of generic force function, $F$ is given as follows:

$$
E(\phi)=\int_{\Omega x} \delta \phi(x) \int_{\Omega y} B(x, y) \bullet F(I(y), \phi(y)) d y d x
$$

$F$ is a generic internal energy measure used to represent local adherence to a given model at each point along the curve. In computing, Lankton's hybrid energy only considers contributions from the points near the contour. By ignoring homogeneity that may arise far away, the ability to capture a much broader range of objects was added. This term ensure that the curve will not changed topology by spontaneously developing new contours, although it still allows for contours to split and merge. For every point selected by $\delta \phi(x)$, Lankton masks the local neighbourhood with $B(x, y)$ to ensure that it is operated only on local image information. Thus, the total contribution of the first term of the energy is the sum of values for every $B(x, y)$ neighbourhood along the zero level set.

Finally, in order to keep the curve smooth, Lankton suggested adding a regularization term. The arch length of the curve is penalized and weights this penalty a parameter, $\lambda$. The final local energy as proposed by Lankton was as followed,

$$
E(\phi)=\int_{\Omega x} \delta \phi(x) \int_{\Omega y} B(x, y) \cdot F(I(y), \phi(y)) d y d x+\lambda \int_{\Omega x} \delta \phi(x)\|\nabla \phi(x)\| d x
$$

By taking the first variation of this energy with respect to $\phi$, the following equation is obtained,

$$
\begin{aligned}
\frac{\partial \phi}{\partial t}(x)=\delta \phi(x) \int_{\Omega y} B(x, y) \cdot \nabla_{\phi(y)} F(I(y), \phi(y)) d y \\
+\lambda \delta \phi(x) \operatorname{div}\left(\frac{\nabla \phi(x)}{|\nabla \phi(x)|}\right)
\end{aligned}
$$

Notice that the only restriction on the internal energy, $\mathrm{F}$ is that its first variation with respect to $\phi$ can be computed. This ensures that nearly all region-based segmentation energies can be put into this framework.

The generic internal energy measure F, can be refer to the global energy in the hybrid energy. The F models the foreground and background as constant intensities represents by their means. Based on Chen-Vese model, the corresponding function $\mathrm{F}$, can be written as

$$
F=\mathrm{H} \phi(y)\left(I(y)-u_{x}\right)^{2}+(1-\mathrm{H} \phi(y))\left(I(y)-v_{x}\right)^{2}
$$

The above $\mathrm{F}$ function is substituted into equation (8) to form a completely hybrid energy. In order to obtains the evolution equation for $\phi$, we calculate the derivative of $\mathrm{F}$ with respect to $\phi(y)$, which can be written immediately as

$$
\nabla_{\phi(y)} F=\delta \phi(y)\left(I(y)-u_{x}\right)^{2}-\left(I(y)-v_{x}\right)^{2}
$$

By inserting this into equation (9), the curvature flow for the localized version in the global energy is captured, thus completing the level set with the hybrid energy minimization algorithm, 


$$
\begin{aligned}
\frac{\partial \phi}{\partial t}(x)= & \delta \phi(x) \int_{\Omega y} B(x, y) \delta \phi(y) \\
& \cdot\left(\left(I(y)-u_{x}\right)^{2}-\left(I(y)-v_{x}\right)^{2)} d y\right. \\
& +\lambda \delta \phi(x) \operatorname{div}\left(\frac{\nabla \phi(x)}{|\nabla \phi(x)|}\right)
\end{aligned}
$$

The $u_{x}$ and $v_{x}$ represents the local means of interior and exterior region respectively, and can be calculate by the following equations

$$
\begin{gathered}
u_{x}=\frac{\int_{\Omega y} B(x, y) \cdot \mathrm{H} \phi(y) \cdot I(y) d y}{\int_{\Omega y} B(x, y) \cdot \mathrm{H} \phi(y) d y} \\
v_{x}=\frac{\int_{\Omega y} B(x, y) \cdot(1-\mathrm{H} \phi(y)) \cdot I(y) d y}{\int_{\Omega y} \chi(x, y) \cdot(1-\mathrm{H} \phi(y)) d y}
\end{gathered}
$$

\subsection{The Liver Level Set Algorithm}

After getting all three elements gear up, the next step is to combine it all into LLS algorithm as shown in Figure 4. This algorithm begins with the input from the pre-processing process, which are the enhanced liver tiff series. The user needs to include three inputs which are the slice number for SLN, ELN and FIN (first initial number). FIN can be chosen by observing the series of input images, and pick an image that contained the largest single liver region. Next, the value of SmN and EmN can be automatically calculated using the equation (1) and (2). The FIN slice is then segmented using the levet set with hybrid energy minimization described above, to obtain an initial binary mask for the looping process. Recalling back the assumption made on the series of CT images are closely related to each other, therefore, the liver boundary in a slice is slightly different from its adjacent slice. This has allowed the use of the segmented liver boundary from the FIN slice as the initial curve for the next slice. Since the FIN slice will be in the middle of the input datasets, the looping process is done in two separated loops. The first loop starts by comparing the current slice, $S_{j}$, number with SLN. If $j$ is bigger then SLN, then the next current slice will be $S_{j-1}$, and the initial curve will be base on previous segmented binary mask as $M_{j}$. This will continue until the value $j$ is equal to SLN. If $\mathrm{j}$ value is equal to SLN, the second loop will begin by comparing the current slice $S_{j}$, number with ELN. Similar with the first loop, if $j$ is smaller then ELN, then the next current slice will be $S_{j+1}$ and the initial curve will be base on previous segmented binary mask, $M_{j}$. This will also continue until the value $j$ is equal to ELN, in which means that all the liver regions have been segmented. 


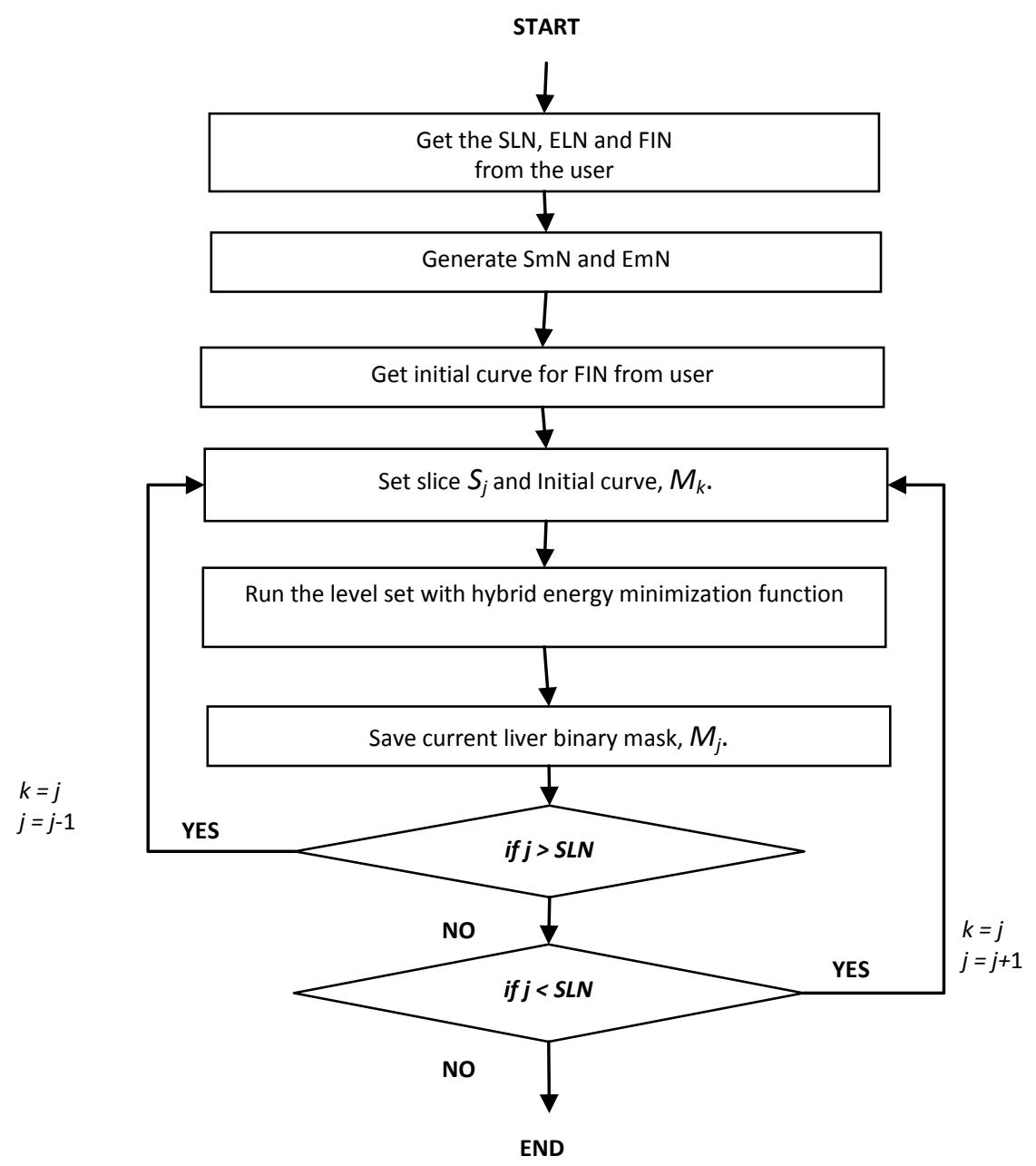

Figure 4: The LLS algorithm

\section{EXPERIMENT AND RESULTS}

In order to demonstrate the strength and limitation of the LLS algorithm, two sets of experiments are performed. The first set of experiments is to validate on the size of liver neighbourhood region while the second set of experiments is to validate on the LLS algorithm segmented images against manually segmented images. The inputs for these experiments are from the range of CT abdominal dataset taken from Heimann et al. (2009).

All the segmentation experiment result generates by the computer will be compared to manual segmentation made by an expert by calculating the liver volume overlapping using the Dice Similarity Coefficient (DSC). The DSC is often used to measure the accuracy of an automatic medical image segmentation algorithm, thus it well suited the liver segmentation. The method is simple and appraises the spatial overlap of two binary images. The coefficient ranges between from 0 for no overlap to 1 for complete overlap. The DSC expressed the overlap of two regions relative to the sum of the two areas. For two regions A (automatic) and $\mathrm{M}$ (manual), the DSC is given by the following equation

$D S C=\frac{2(M \cap A)}{M+A}$

\subsection{Liver Local Neighbourhood Region Validation}

In the liver neighbourhood region validation, the experiment begins by testing the LLS algorithm with various size of liver neighbourhood region. As the input for this experiment, 20 series of enhanced tiff images obtain after the pre-processing process are used. And the output for this experiment will be compare with an expert manual segmentation in DSC percentage. Next, the significant of using the generalization factor of Gfs and Gfe 
using the equation (1) and (2) is validated. The results of these automatic generated values are compared to manually generated values, where their error rates are calculated.

\subsubsection{Comparison with Various Region Sizes}

In the hybrid energy minimization technique, the size of local neighbourhood region is an important parameter and it should be chosen based on the scale of the object of interest with the presence and proximity of surrounding clutter. With the same initialization curve, it is possible to obtain two different results. Therefore by varying the size of liver local neighbourhood, we can find the optimum for liver neighbourhood region. The size of local liver neighbourhood region is defined as RL (radius length). The experiment starts by giving a fixed value of RL, ranging from 5 to 25 in the increment of 5. The initial curve for each current slice is taken from previous slice of the manually segmented liver region. This is to control so that the initial curve placement would not be a contributing factor that will effect segmentation the result. The number of iteration for each slice is set to 50 times. Figure 5 and 6 illustrate the result effect of different radius length.

In Figure 5, the example is taken from a slice that has been categorized in stage 1, that has a clear boundary of liver region separating the rib muscle with the absent of adjacent organs such as kidney and gallbladder. The Figure 5 (a) shows the slice image and its initial curve, which is slightly larger from the current liver region. Figure 6 (b) shows the segmentation result with the local neighbourhood region set with RL as 5. The red liver contour has successfully propagated and shrinks toward the liver boundary on the left side. However, the red liver contour fails to capture the liver boundary on the bottom site. Figure 5 (c) with RL value as 10 , illustrates the bottom liver region has correctly recognized. However it also illustrates that the right side of the liver has failed to be segmented. Meanwhile, in Figure 5 (d) and (e) with RL as 15 and 20 respectively, shows the best segmentation results, where all the liver boundaries has successfully extracted. Meanwhile, the largest RL values, 25, give a leaking result as shown in Figure 5 (f). Therefore, in this example, the smallest (5, 10) and the largest (25) RL values give unsatisfying segmentation result and the intermediate size of 15 and 20 give the desirable segmentation result.

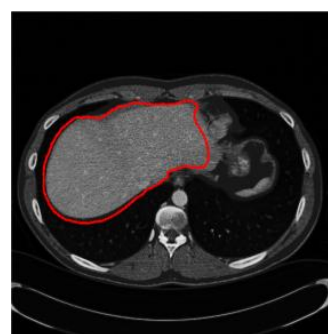

(a) initial curve

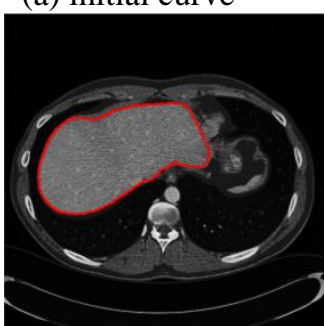

(d) $\mathrm{RL}=15$

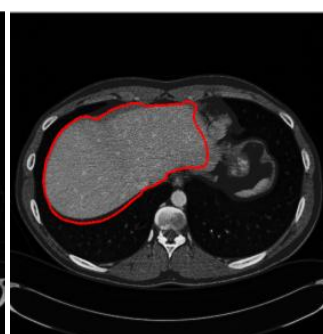

(b) $\mathrm{RL}=5$

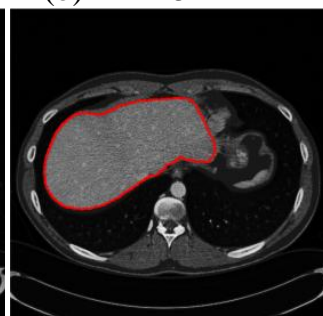

(e) $\mathrm{RL}=20$

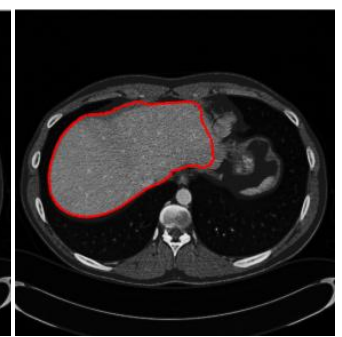

(c) $\mathrm{RL}=10$

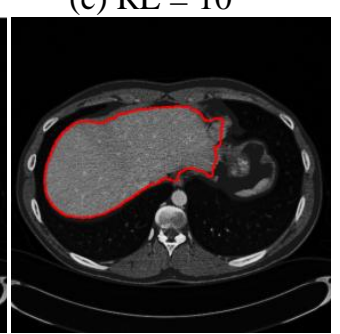

(f) $\mathrm{RL}=25$

Figure 5: Segmentation result of a patient slice in stage 1 with different RL.

For the next example, a slice is taken from stage 2, which contains a liver region surrounded with several artefacts such as the ribs bone and ribs muscle. Figure 6 give a different segmentation result. The smallest radius, RL as 5, derive a correct segmentation result, while the intermediate and large give out a false segmentation result. Figure 6 (c) shows that the liver boundary starts to leak towards the liver tumours. The larger RL value has caused a larger leaking toward the tumour region. 


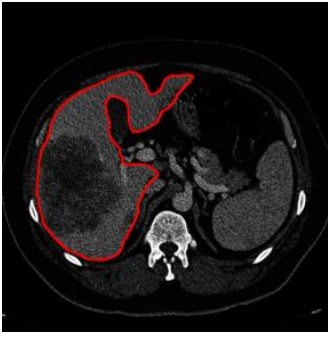

(a) initial curve

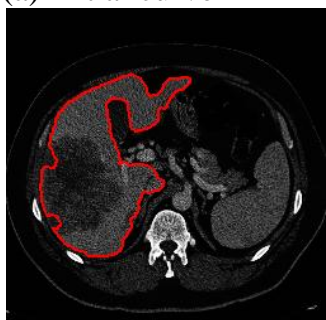

(d) $\mathrm{RL}=15$

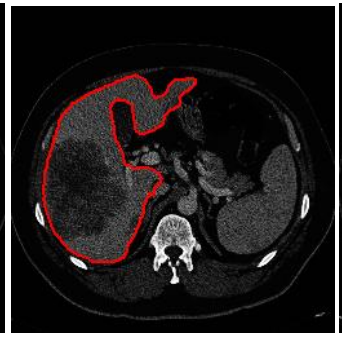

(b) $\mathrm{RL}=5$

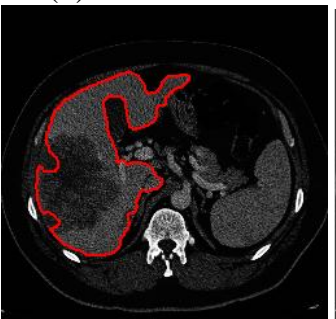

(e) $\mathrm{RL}=20$

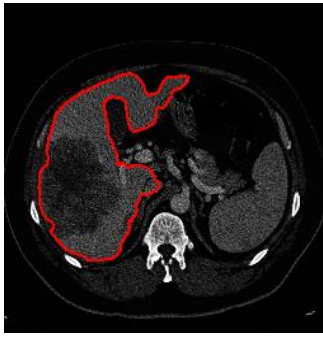

(c) $\mathrm{RL}=10$

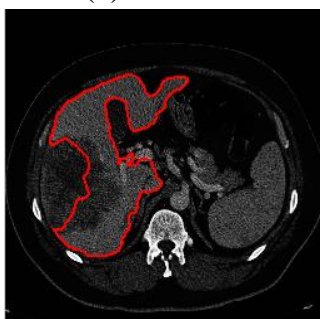

(f) $\mathrm{RL}=25$

Figure 6: Segmentation result of a patient slice in stage 2 with different RL.

In order to check the reliability of the suggested RL values, a frequency graph is generated that take the DCS percentage for every patient with the best LR value for each stage. The frequency graph in Figure 7 shows reading of DSC percentage is in between $83 \%$ to $87 \%$ for stage 1 . Meanwhile in stage 2, the DSC percentage is between $80 \%$ to $82 \%$ and stage 3 the DSC percentage value is in between $85 \%$ to $88 \%$. The overall graph shows a stable frequency for stage 2 and 3 but a slightly jagged for stage 1 . These due to the fact that some of the liver boundary in stage 1 has close intensity with muscle rib, causing a small amount of leakages, therefore causing a slightly lower reading of DSC percentage. Stage 2 also give a lower reading of correctness due to multiple artefacts in stage 2 , but the overall still give a stable results with more than $80 \%$ of DSC percentage.

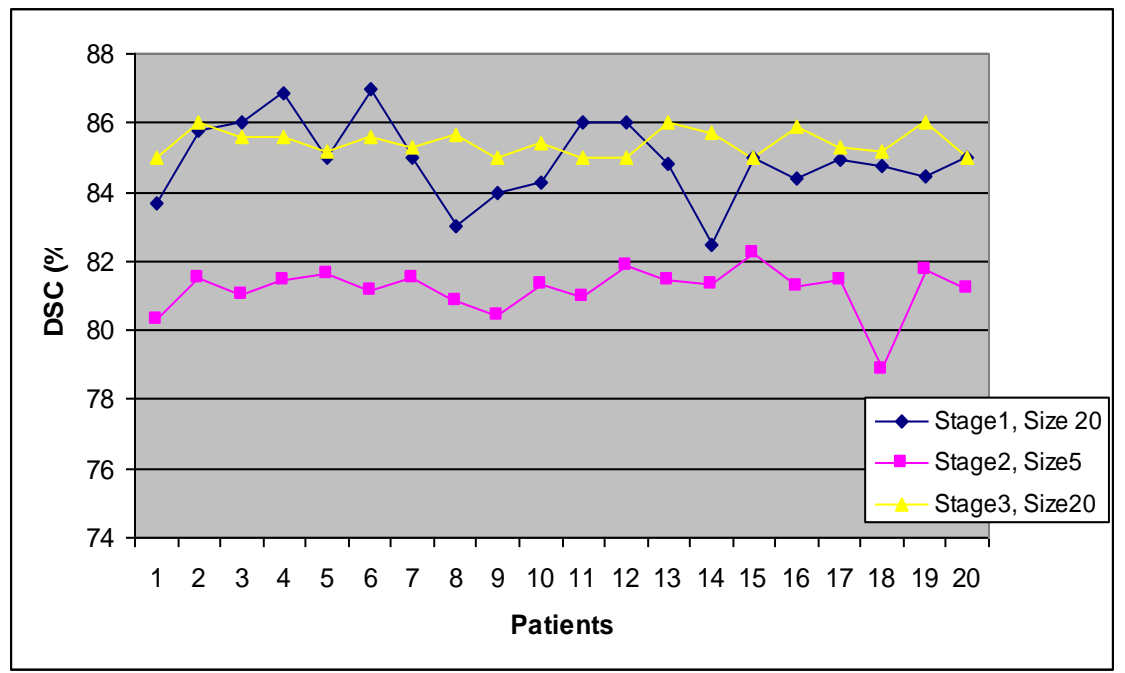

Figure 7: The frequency graph for stage 1, 2 and 3 (20 patients)

\subsubsection{Comparison on Manually and Computer Generated SmN}

The second experiment is to compare the manually tagged $\mathrm{SmN}$ with the generated $\mathrm{SmN}$ using the equation (1). The same datasets is used for generating the generalization factor, consisting 20 patients. Overall, the error is between +- 5 slices with an error rate of $2.5 \%$ for an average slice 196 images as illustrates in Figure 8, where its shows clearly that both lines are almost overlapping each other. An exception can be seen with patient number 5 with an error margin of 17 slices. The reason for this large error is that this patient unable to lie down normally during the CT scan process, causing the series of images capture trough CT scan to be distorted form normal reading. 


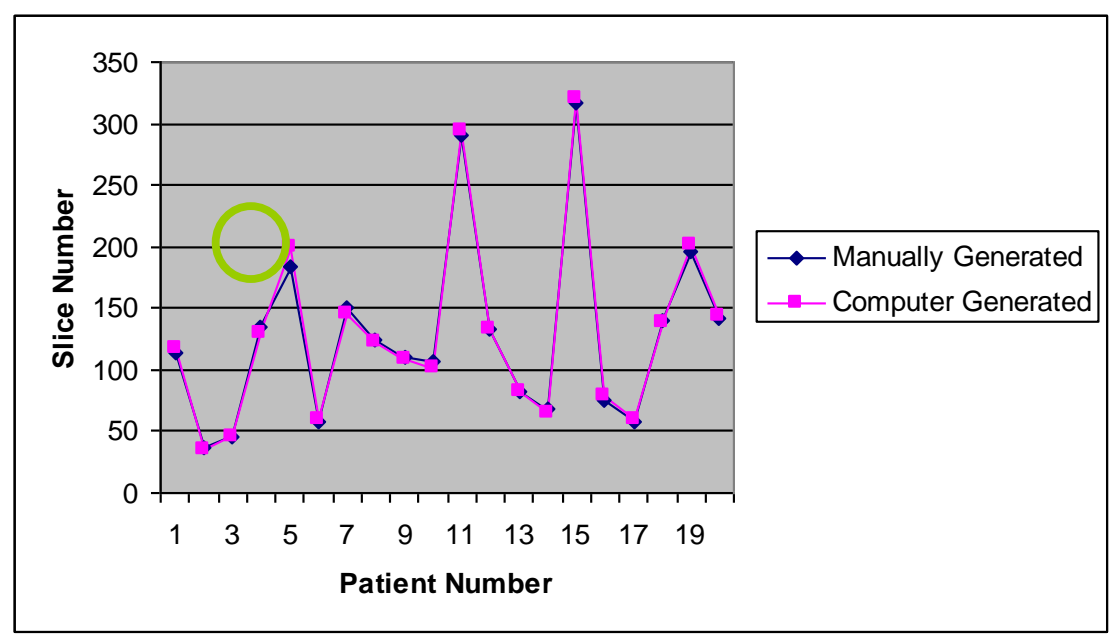

Figure 8: A line graph for error in $\mathrm{SmN}$ generated for 20 patients

\subsubsection{Comparison on Manually and Computer Generated EmN}

The third experiment is to compare the manually tagged EmN with the computer generated EmN using the equation (2). Similar with $\mathrm{SmN}$ experiment, the calculated errors are between +-5 slice with an error rate of $2.5 \%$ from an average 196 images. Figure 9 illustrates the line graph, where the problem can be seen with patient number 5 showing high differences between the lines.

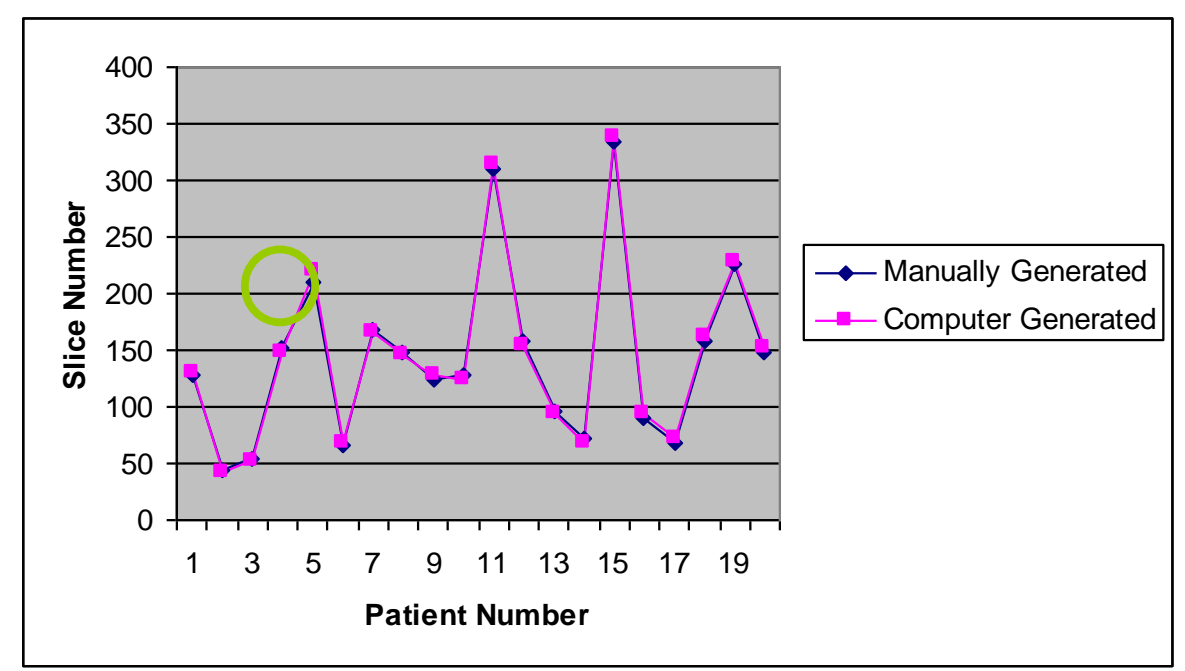

Figure 9: A line graph for error in $\mathrm{EmN}$ generated for 20 patients

\subsection{The LLS Algorithm Validation}

Validation for LLS algorithm is conducted in two parts of experiments. The first experiment uses a fixed size liver neighbourhood region, while the second experiment uses variable size of liver neighbourhood region, in three stages. The value of RL for the first experiment is that 17 . For the second phase, the RL value is 17 for stage 1 and 3, while in stage 2, the RL value is 5 . The inputs for these experiments are nine patients that contain multiple disconnected liver regions. The final segmentation results are compared with manual segmentation using DSC percentage as shown in table 1. The first experiment give a respective result of an average 85.54 percent in DSC value, while the second experiment showed an improvement with an average of $87.62 \%$ in DSC value. The percentage of improvements for patient number $2,3,5,6$ and 9 were more than $1.5 \%$. 
Table 1. DSC percentage between two part experiments for nine patients

\begin{tabular}{|l|l|l|l|l|}
\hline Patient & $\begin{array}{l}\text { Number } \\
\text { disconnected regions }\end{array}$ & $\begin{array}{l}\text { First Experiment } \\
\text { DSC [\%] }\end{array}$ & $\begin{array}{l}\text { Second Experiment } \\
\text { DSC [\%] }\end{array}$ & Improvement [\%] \\
\hline 1 & 2 & 87.98 & 89.02 & 1.17 \\
\hline 2 & 3 & 82.45 & 85.34 & 3.39 \\
\hline 3 & 5 & 79.78 & 82.73 & 3.57 \\
\hline 4 & 1 & 91.43 & 92.81 & 1.49 \\
\hline 5 & 2 & 81.74 & 85.02 & 3.86 \\
\hline 6 & 3 & 85.82 & 88.92 & 3.49 \\
\hline 7 & 2 & 89.12 & 90.04 & 1.02 \\
\hline 8 & 2 & 92.55 & 92.63 & 0.09 \\
\hline 9 & 4 & 79.03 & 82.10 & 3.74 \\
\hline AVERAGE DSC & $\mathbf{8 5 . 5 4}$ & $\mathbf{8 7 . 6 2}$ & $\mathbf{2 . 4 2}$ \\
\hline
\end{tabular}

\section{CONCLUSION}

In this paper, the problem highlighted is how to segment the liver regions that are disconnected in a single slice automatically. Therefore, LLS algorithm has been proposed to carter these problems. In the LLS algorithm a dynamic local neighbourhood regions has been proposed by examining the characteristic of liver CT scan images. The liver local neighbourhood regions has been divided into three stages, with SLN, ELN, SmN and EmN slices are recognized as the mark images. By normalizing these mark images, we obtain the generalization factor, Gfs and Gfe, from the average of normalized $\mathrm{SmN}$ and $\mathrm{EmN}$. In addition to this, the two generalization factor equations are made allowing the value of $\mathrm{SmN}$ and $\mathrm{EmN}$ to be obtained automatically using the Gfs and Gfe values. The experiments result shows that by dividing the liver into three stages and given it different sizes of liver local neighbourhood regions, there is an overall improvement of $2.4 \%$ percent DSC reading. The proposed LLS algorithm has successfully segmented the liver regions whether connected or disconnected with an average DCS percentage of $87 \%$.

However, the limitation with the LLS algorithm is on its reliability of the GFe and Gfs variable to give the different neighbourhood sizes based on three stages. .The improvement suggested is deriving the neighbourhood sizes based on the overall liver volume sizes automatically, rather than these stages. Plus, another disadvantage is that choosing the wrong first slice, would lead to wrong segmentation result. It will get worst, since the wrong segmented information will be inherited to the next slice causing even more wrong segmentation results

\section{REFERENCES}

Areste R., Yongyi Y. and Jiang H (2005). An Image Enhancement Procedure for 3D Visualization of Liver CT Data. In Image Analysis and Interpretation, Proceedings of IEEE Southwest, Denver.

Bae K.T., Giger M.L., Chen C.T. and Kahn C.E. Jr. (1993). Automatic segmentation of liver structure in CT images. Journal of Medical Physics. 20(1):71-78.

Gao L., Heath, D.G. and Fishman, E.K. (1998). Abdominal image segmentation using three-dimensional deformable models. Journal of Investigative Radiology. 33 (6):348-355.

Gao L., Heath D.G., Kyszyk B.S. and Gishman E.K. (1996). Automatic liver segmentation technique for threedimensional visualization of CT data. Journal of Radiology. 201: 359-364.

Kass M., Witkin A. and Terzopoulos D. (1988). Snakes: active contour models. Journal of Computer Vision.1(4): 321-331.

Kobashi M. and Shapiro L. (1995). Knowledge-based organ identification from CT images. Journal of Pattern Recognition. 28(4):475-491.

Lamecker H., Lange T. and Seebass M. (2004). Segmentation of the liver using a 3D statistical shape model; ZIBReport 04-09, Berlin. 
Lankton S., Delphine N., Anthony Y., and Allen T. (2007) Hybrid Geodesic Region-Based Curve Evolutions for Image Segmentation. In Proceedings of SPIE Medical Imaging.

Lee J., Kim N., Seo J.B., W H.J., Shin Y.M. and Shin Y.G. (2007). Efficient liver segmentation exploiting level set speed images with $2.5 \mathrm{D}$ shape propagation. In: 3D Segmentation in the Clinic: A grand Challenge, pp. 189-196.

Lim S.J., Jeong Y.Y., Lee C.W. and Ho Y.S (2004) Automatic segmentation of the liver in CT images using the watershed algorithm based on morphological filtering. In Medical Imaging, Proceedings of SPIE.

Lim S.J., Jeong Y.Y. and Ho Y.S. (2006). Automatic liver segmentation for volume measurement in CT images. Journal of Visual Communication and Image Representation. 17(4): 860-875.

Lim S.J., Jeong Y.Y. and Ho Y.S. (2005) Segmentation of the liver using the deformable contour method on ct images, In Medical Imaging, Proceedings of SPIE.

Liu F., Zhao B, and Kijewski P.K. (2005). Liver segmentation for CT images using gvf snake. Journal of Medical Physic. 32(12): 3699-3706.

Pan S., and Dawant B. M. (2001) Automatic 3D egmentation of the liver from abdominal CT images: a level set approach. Proceeding of SPIE, vol. 4322 pp. 128-138

Pham D.L., Xu, C. and Prince, J.L. (2000). A survey of current methods in medical image segmentation. Journal of Annual Review of Biomedical Engineering. 2:315-337.

Shimizu A., Ohno R., Ikegam, T., Kobatake H., Nawano S. and Smutek D. (2007). Multi-organ segmentation in three dimensional abdominal CT images. Journal of Computer Assisted Radiology and Surgery. 1(7):7678.

Sulaiman P.S., Rahmat R.W., Mahmod R. and Rashid A.H. (2008) A Liver Level Set (LLS) Algorithm for Extracting Liver's Volume Containing Disconnected Regions Automatically. In IJCSNC Vol. 8 No. 12 pp. 246-252.

Heimann T, van Ginneken B, Styner MA, Arzhaeva Y, Aurich V, Bauer C, Beck A, Becker C, Beichel R, Bekes G, Bello F, Binnig G, Bischof H, Bornik A, Cashman PM, Chi Y, Cordova A, Dawant BM, Fidrich M, Furst JD, Furukawa D, Grenacher L, Hornegger J, Kainmüller D, Kitney RI, Kobatake H, Lamecker H, Lange T, Lee J, Lennon B, Li R, Li S, Meinzer HP, Nemeth G, Raicu DS, Rau AM, van Rikxoort EM, Rousson M, Rusko L, Saddi KA, Schmidt G, Seghers D, Shimizu A, Slagmolen P, Sorantin E, Soza G, Susomboon R, Waite JM, Wimmer A, Wolf I. (2009) Comparison and Evaluation of Methods for Liver Segmentation from CT datasets, IEEE Transactions on Medical Imaging, volume 28, number 8, pp. 12511265.

Zhou X., Kitagawa T., Okuo K., Fujita H., Yokoyama R., Kanematsu M., and Hoshi H. (2005). Construction of a probabilistic atlas for automated liver segmentation in non-contrast torso CT images. Proceedings of CARS. 